\title{
Probability Density FunCtions of the PACKET LENGTH FOR COMPUTER NeTWORKS WITH BIMODAL TRAFFIC
}

\author{
Ewerton R. S. Castro ${ }^{1}$, Marcelo S. Alencar ${ }^{2}$ and Iguatemi E. Fonseca ${ }^{3}$ \\ ${ }^{1}$ Federal Institute of Paraíba, Patos, Brazil \\ ewerton.castro@ifpb.edu.br \\ ${ }^{2}$ Federal University of Campina Grande, Iecom, DEE, Brazil \\ malencar@dee.ufcg.edu.br \\ ${ }^{3}$ Federal University of of Paraíba, Brazil \\ iguatemi@ci.ufpb.br
}

\begin{abstract}
The research on Internet traffic classification and identification, with application on prevention of attacks and intrusions, increased considerably in the past years. Strategies based on statistical characteristics of the Internet traffic, that use parameters such as packet length (size) and inter-arrival time and their probability density functions, are popular. This paper presents a new statistical modeling for packet length, which shows that it can be modeled using a probability density function that involves a normal or a beta distribution, according to the traffic generated by the users. The proposed functions has parameters that depend on the type of traffic and can be used as part of an Internet traffic classification and identification strategy. The models can be used to compare, simulate and estimate the computer network traffic, as well as to generate synthetic traffic and estimate the packets processing capacity of Internet routers
\end{abstract}

\section{KEYWORDS}

Packet-switching networks, Measurement, Modeling, Statistical methods \& Packet length.

\section{INTRODUCTION}

The evolution of the Internet in the last few years has been characterized by changes in the way users behave, interact and utilize the network. The availability of broadband connections, particularly those based on cable TV and ADSL technologies, and new applications and dynamics of the Internet traffic, such as P2P applications and video sharing, have caused changes in various network management activities, such as capacity planning and provisioning, traffic engineering, application performance, anomaly detection and pricing. The correct identification of user applications may give valuable information for network operators to develop new traffic engineering policies and for providers to offer services based on user demand. Diagnosing anomalies is critical for both network operators and end users in terms of data security and service availability. Anomalies, such as the ones produced by worms or Denial of Service (DoS) attacks, may have unexpected behavior and may cause significant changes in the network traffic. Besides that, knowledge of the applications used by the subscribers may be of interest for application-based accounting and charging or even for the Internet Service Provider (ISP) to offer new products [1]-[6].

Characterization of Internet traffic is an important issue for telecommunications networks [7]. The literature presents several traffic identification techniques, and those using packet length (size) are important strategies [1]-[24]. The research usually involves experimental measurements $[13],[14],[17]-[23]$, because this information can be used to design and estimate the network 
infrastructure and application attributes. Li Bo, for example, indicates that the packet size (or length) distribution can be used to identify many Transport Control Protocol (TCP) applications [18]. Alfonso Iacovazzi reports that statistical traffic classification is possible based on some features of the Internet Protocol (IP) flow and that the packet length is a key feature to classify the application level content of a packet flow and that this classification can be useful for security policies, traffic filtering and support to the quality of service mechanisms [7]. Mushtaq mentioned that the probability density function and the cumulative distribution function can be used to design, control, manage, interpolate and extrapolate the network traffic. It enables to develop professional simulators and fast, efficient and robust algorithms for the optimization of computer and communication systems, along with applications [22].

Therefore, the analysis and characterization of those parameters is important to design new methods for traffic identification and classification. Traffic classification based on packet length was attempted by some authors in recent papers [1]-[5]. The main contribution of this paper is to propose a new probability density function for the packet length which can be used to identify and classify the Internet traffic. This paper also summarizes results from the literature on packet size distribution. Using these results, mathematical models for the packet distribution are presented and compared with actual measurements of packet size. The analysis of the results suggests that the model can be used to estimate the distribution of packet traffic in computer networks. Besides, this investigation shows that it is possible to relate parameters of the proposed distribution function with the traffic type or network application.

The remaining of the paper is organized as follows. Section 2 presents mathematical models to estimate the length distribution of the packets. Section 3 compares the measured values with the proposed mathematical models. Section 4 shows a summary of applications. Section 5 presents the conclusions of the paper.

\section{Mathematical Models}

The usual configuration for a Local Area Network (LAN) with Internet access is shown in Figure

1. Using this configuration some mathematical models for the packet length in computer networks can be derived. 


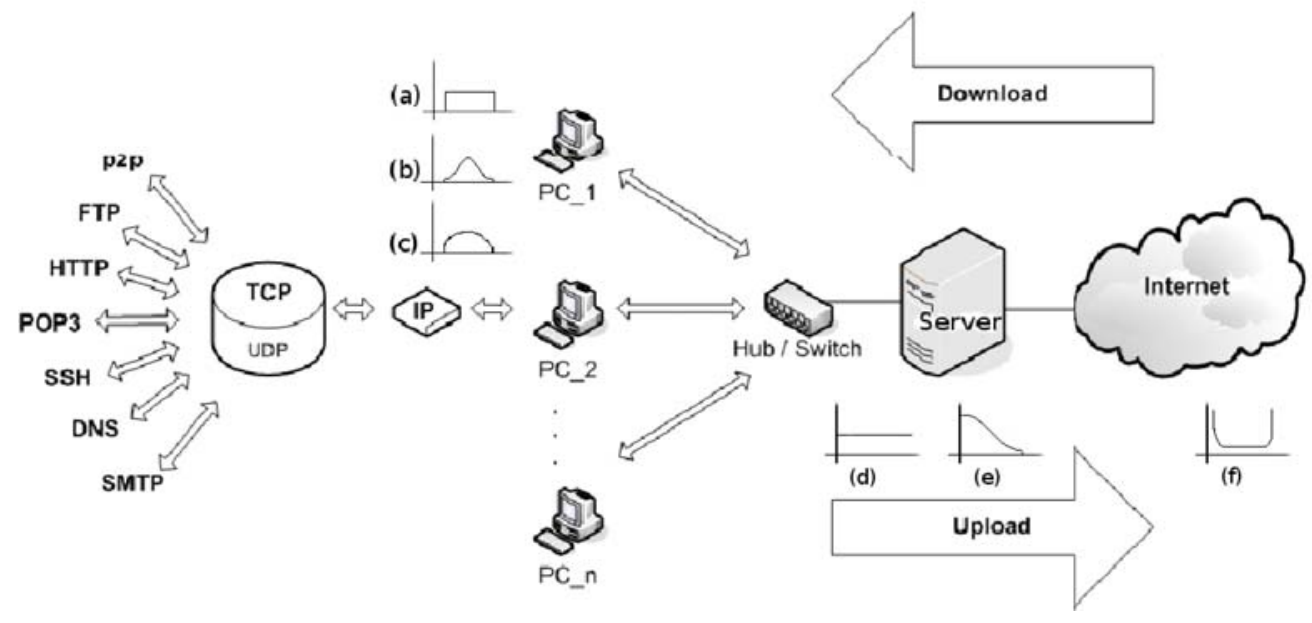

Figure 1. Packet aggregation by the network server

The packet length was measured between the network server and the Internet connection, as illustrated in Figure 1, to obtain the packet length relative frequency distribution. The results are discussed in Section 3 and show that the packet length has a bimodal traffic distribution. Similar results are found in [13]-[23]. Using the measured values, one can derive two equations to describe this bimodal traffic distribution, which can be obtained using a mathematical procedure presented in this paper. In the absence of a priori information, the Uniform (Figure 1a) is usually considered for the packet length generated by the computers (users). The Normal (Figure 1b) and Beta (Figure 1c) distributions are considered when additional information can be obtained. The discussion and mathematical analysis are presented in the Section 2.1, for the Uniform and the Normal distribution, and in the Section 2.2 for the Beta distribution.

\subsection{Model based on the Normal distribution}

Consider that the traffic produced by a computer (user) presents, originally, a uniform distribution (Figure 1a) for the packet length, as shown in Figure 2a. When the data traffic flows through the aggregation point (router, gateway or server) it suffers a non-linear transformation (Figure 1d), which produces a bimodal distribution [13]-[23] (Figure 1f). Figure 2b shows this non-linear transformation at the aggregation point, which generates the bimodal distribution (Figure 2c), in accordance with the published results.

However, considering that the packets are produced by several computers one can invoke the Central Limit Theorem, and infer that the aggregate traffic has a Gaussian distribution (Figure 1b), as shown in Figure 3a [27]. Therefore, when the data traffic flows through the aggregation point, it suffers a non-linear transformation (Figure 1e) and the network servers group the packets (bytes) according to the adopted protocol (IP, ICMP, TCP or UDP), as depicted in Figure 1, and this produces a non-uniform distribution for the packet size, as shown in Figure 3c (or Figure 1f). The probability density function transformation (illustrated in Figure 3b), at the aggregation point can be obtained using

$$
\ell=\frac{\cos x+M}{2}
$$


in which, $\mathrm{M}$ is the normalized MTU (1500 bytes/1500 bytes), $\ell$ is the output that represents the packet length (size) and it is function of the $\mathrm{x}$, the input random variable. This is the traffic generated by the user and it is not known previously. Equation (1) is responsible for the nonlinear transformation that appears in the following.

Rewriting $\mathrm{x}$ as a function of $\ell$, gives

$$
x=\arccos (2 \ell-M)
$$

in which $\ell$ is the non-linear transformation used in Figure $3 \mathrm{~b}$ and

$$
p_{N}(\ell)=\frac{p_{L}(x)}{\left|\frac{d \ell}{d x}\right|}=\frac{p_{L}(x)}{\frac{1}{2} \sqrt{1-(2 \ell-M)^{2}}}
$$

in which $p_{N}(\ell)$ is the probability density function of a packet length after the aggregation point [27].

Considering that the traffic produced by a single computer presents a Normal distribution for the packet length, then



(a)

Figure 2. Non-linear transformation of probability density function. (a) Uniform distribution, (b) Nonlinear transformation and (c) Probability density function. 


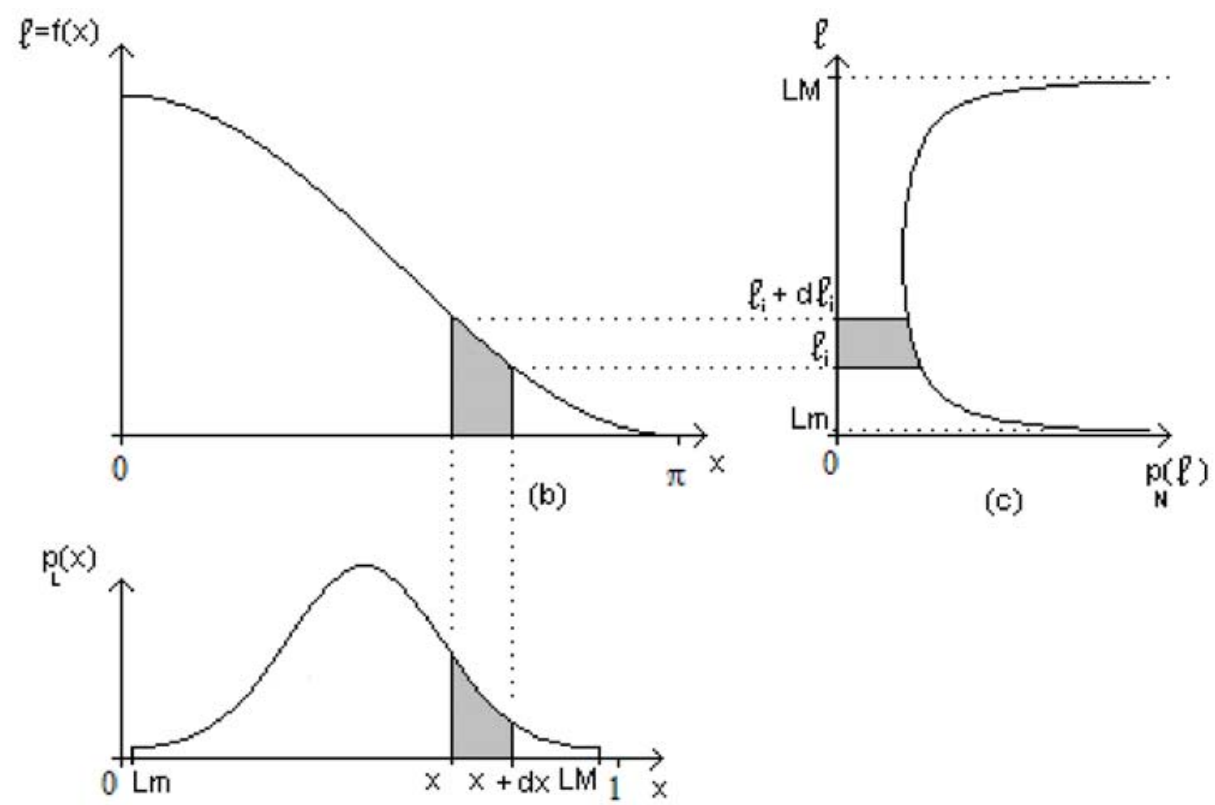

(a)

Figure 3. Non-linear transformation of probability density function. (a) Normal distribution. (b) Nonlinear transformation and (c) Probability density function.

$$
p_{L}(x)=\frac{1}{\sigma \sqrt{2}} e^{-\frac{(x-m)^{2}}{2 \sigma^{2}}}
$$

in which, $\mathrm{x}$ is given by Equation (2), $\mathrm{E}[\mathrm{x}]=\mathrm{m}$ is the mean and $\sigma^{2}$ is the variance.

Using equations (3) and (4) with $\mathrm{m}=0$, the following equation is proposed to model the network traffic distribution

$$
p_{N}(\ell)=\frac{2}{\sigma \sqrt{2 \pi}} \frac{e^{-\frac{(\arccos (2 \ell-M))^{2}}{2 \sigma^{2}}}}{\sqrt{1-(2 \ell-M)^{2}}}
$$

in which, $p_{N}(\ell)$ is the probability density function of the packet length at the output of the aggregation point, $\ell$ and $\sigma$ are parameters of the distribution function related to the traffic type.

Notice that $\sigma^{2}$ is the variance of the Normal distribution, which is related with the traffic generated by the computers [27]. This is an important parameter and, as seen in the Section 5, may be associated with the type of traffic in the network. Paper [27] presents the comparison between $p_{N}(\ell)$ and experimental measurements. 


\subsection{Model based on the Beta distribution}

The Normal distribution (Figure 3a) is useful, but has a long tail which does not properly adjust to the limited range of packet length defined by the Internet transport protocols.

The Beta distribution provides better approximations, that match the practical results, considering that the limited range of the random packets, Figure 4a, and Figure 1c. This leads, after the nonlinear transformation (Figure 1e or Figure $4 \mathrm{~b}$ ), to the new model discussed in the following, originally proposed by Castro et al [28]. The initial conditions to derive the mathematical packet length model, to obtain the probability density function presented in Figure 4, are:

- Consider that $\mathrm{x}$ is a random variable, with $0 \leq x \leq 1$;

- The minimum and maximum normalized packet length are $L_{m}$ and $L_{M}$ defined by:

$$
\begin{gathered}
0 \leq L_{m}<1 \\
\text { e } 0 \leq L_{M}<1, L_{m}<L_{M}
\end{gathered}
$$

the parameters are the ratio between the minimum (maximum) packet length, in terms of bits or bytes that can be sent through a network interface, and $N_{\operatorname{Max}} . N_{\operatorname{Max}}$ is the maximum number of bits or bytes that can be send through a network interface in a time interval $t$.

Definition 1: consider that $\ell(\mathrm{x})$, or simply $\ell$, is the random variable that represents the normalized packet length sent through a network interface in a time interval $t$, and that it is possible to express $\ell$ by

$$
\ell=L_{M}-\left(\frac{L_{M}-L_{m}}{2}\right)\left[\cos \left(\frac{\pi x}{n}\right)+1\right], n \in N
$$

and from Equation (8), one can obtain the random variable $\mathrm{x}$ as a function of the packet length $\ell$

$$
x=\frac{1}{\pi} \arccos \left[2\left(\frac{L_{M}-\ell}{L_{M}-L_{m}}\right)-1\right]
$$

Equation (8) or (9), presented in Figure 4b, represent the non-linear transformation of the Beta distribution and that transformation gives the bimodal distribution illustrated in Figure 4c [13][23] illustrated in Figure 4c. This equation is obtained from Equation (1) [27] after some simulations and curve fitting [26]. 


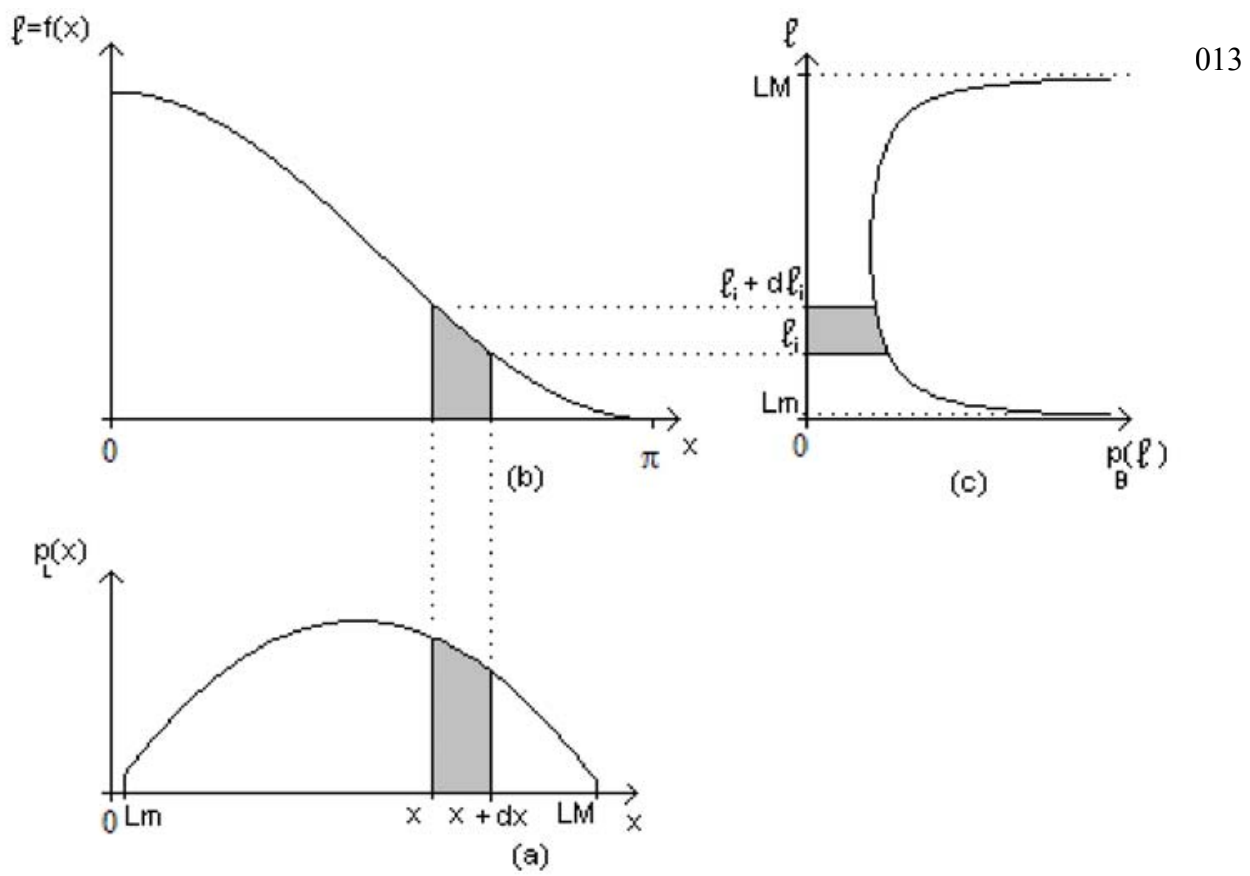

Figure 4. Aggregated packet length non-uniform distribution. (a) Beta distribution $\mathrm{B}(2,2)$, Non-linear transformation and (c) Probability density function.

Initially, the possibility of obtaining a consistent probability density function using curve fitting by software, as for example Matlab, was investigated. A few models were obtained, but they had several tuning parameters (the minimum number was four parameters, and the approximation was poor). With this number of parameters is it difficult to associate each of them with the use of the probability density function in methods for traffic identification or classification. Therefore, the mathematical approach was preferred.

For the Ethernet network standard the Maximum Traffic Unit (MTU) is $N_{\text {Max }}=1500$ bytes. The maximum packet length that can be sent through a network interface is 1492 bytes, because eight bytes are used in the LLC header [25]. This gives $L_{M}=\left(\frac{1492}{1500}\right) \quad 0.9946$, but for computation purposes one considers $L_{M}=\left(\frac{1491}{1500}\right)=0.994$. The minimum packet length is 28 bytes ( 20 bytes for the IP header +8 bytes used in the LLC header), that can be sent through a network interface, this means $L_{m}=\left(\frac{28}{1500}\right) \quad 0.0186$ or $L_{m}=\left(\frac{27}{1500}\right)=0.018$ for computation purposes. Other values for $L_{m}$ are also possible depending on how the protocols are combined.

The following formula, obtained from the non-linear transformation (8), with the bimodal traffic distribution, is proposed to model the packet length probability density function after the aggregation point. 


$$
\begin{aligned}
& p_{B}(\ell)=\frac{1}{\pi \sqrt{\left(\frac{L_{M}-L_{m}}{2}\right)^{2}-\left[\ell-\left(\frac{L_{M}+L_{m}}{2}\right)\right]^{2}}\left[\frac{\Gamma(\alpha+\beta)}{\Gamma(\alpha) \Gamma(\beta)}\right]} . \\
& \left\{\frac{1}{\pi} \arccos \left[2\left(\frac{L_{M}-\ell}{L_{M}-L_{m}}\right)-1\right]\right\}^{\alpha-1} \cdot\left\{1-\frac{1}{\pi} \arccos \left[2\left(\frac{L_{M}-\ell}{L_{M}-L_{m}}\right)-1\right]\right\}^{\beta-1}
\end{aligned}
$$

$\mathrm{ou}$

$$
p_{B}(\ell)=\frac{1}{\pi \sqrt{A_{1^{2}}-A_{2^{2}}}} \cdot \frac{1}{B(\alpha, \beta)}\left[\frac{\Gamma(\alpha+\beta)}{\Gamma(\alpha) \Gamma(\beta)}\right] \cdot(x)^{\alpha-1}(1-x)^{\beta-1}
$$

in which, $\mathrm{x}$ is given by (9), $A_{1}=\left(\frac{L_{M}-L_{m}}{2}\right)$ is the midpoint of the interval, $A_{2}=\left[\ell-\left(\frac{L_{M}+L_{m}}{2}\right)\right]$ is the mean, $\mathrm{B}($.$) is the Beta function, \Gamma($.$) is the Gama function, \ell$ is a random variable that represents the packet length (or size) after the aggregation point, $p_{B} \ell$ is the probability density function of a packet length $\ell$ obtain from the Beta distribution, $\alpha$ nd $\beta$ are parameters of the distribution function related to the traffic type, $L_{M}$ is the maximum packet length, $L_{m}$ is the minimum packet length, $L_{m}>0, L_{M}>0$ and $L_{m}<\ell<L_{M}$.

The cumulative distribution function of the packet length is

$$
P_{L}(\ell)=I_{x}(\alpha \alpha \beta)
$$

is modeled as a regularized incomplete Beta function, in which, $I_{0}(\alpha \alpha \beta)=0, I_{1}(\alpha \phi \beta)=1$ and $\mathrm{y}=\mathrm{x}(\ell)$ is given by

$$
x=\frac{n}{\pi} \arccos \left[2\left(\frac{L_{M}-\ell}{L_{M}-L_{m}}\right)-1\right]
$$

One can obtain Equation (13) as a function of the packet length $\ell$, given by Equation (8), with $\mathrm{n}=1$.

\section{Comparison Results}

This section shows a comparison between the proposed models and some measurements. The measurements are obtained from: backbones with different bit rates [20], [21], ISP with Wi-Fi access [14], data traffic between cities (Chicago and Seattle) [21], and measurements made by the authors [27]-[30].

The proposed probability density function is compared with measurements presented in several articles [14]-[16], [20], [21], which were obtained from different networks scenarios, as, for 
example, backbones with different bit rates [20], [21], ISP with Wi-Fi access [14], and data traffic between cities (Chicago and Seattle) [21].

\subsection{Tafvelin measurements}

A comparison between the measurements found in the literature and results obtained from the proposed models for the cumulative distribution function of the packet length is shown in the following figures and tables.

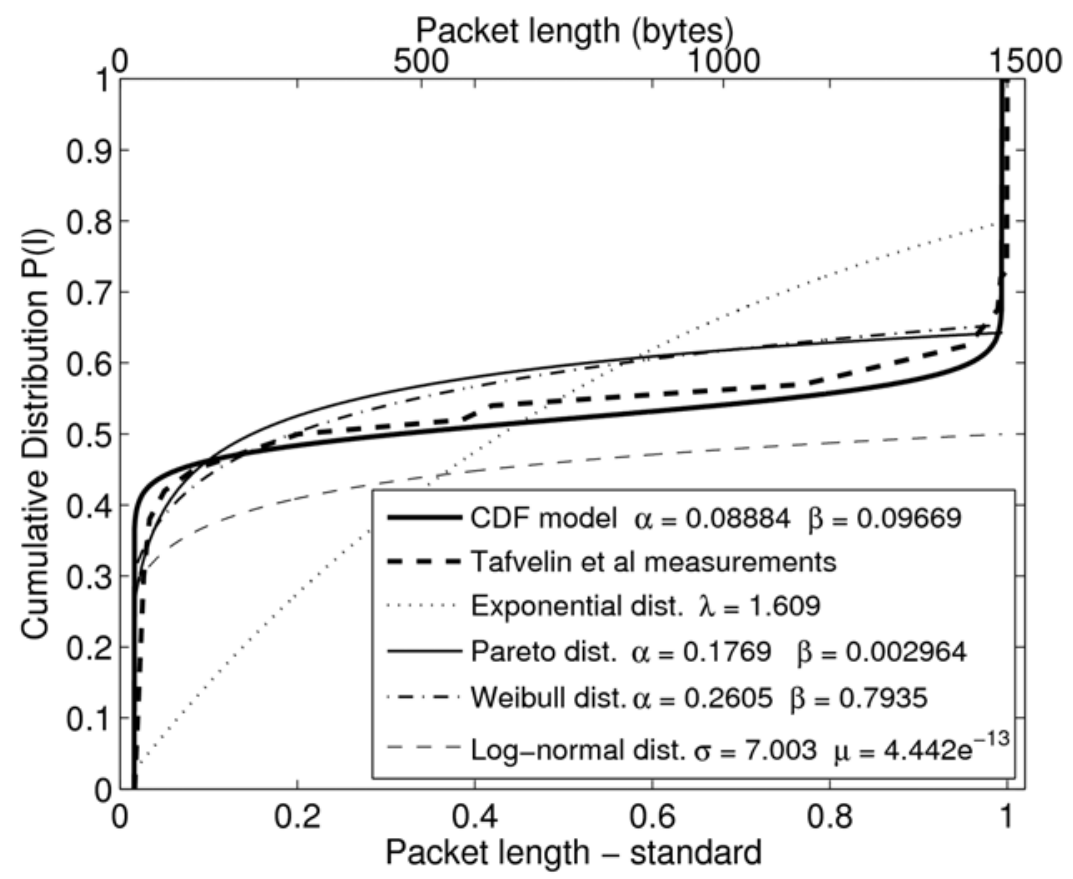

Figure 5. Tafvelin [15] measurements versus $P_{B}(\ell)$ function and other cumulative distributions.

Table 1. Tafvelin [15] measurements versus, $P_{B}(\ell)$ and other cumulative distributions.

\begin{tabular}{|l|l|l|l|l|l|l|}
\hline Dist. & Par.1 & Par.2 & SSE & RMSE & RS & ARS \\
\hline Exp & $\lambda=1.6$ & - & 0.59 & 0.21 & 0.17 & 0.17 \\
\hline Log & $\mu=4 \mathrm{e}-13$ & $\sigma=7.0$ & 0.54 & 0.19 & 0.24 & 0.24 \\
\hline Par & $\alpha=0.18$ & $\beta=0.003$ & 0.03 & 0.05 & 0.87 & 0.86 \\
\hline Wei & $\alpha=0.26$ & $\beta=0.79$ & 0.02 & 0.05 & 0.89 & 0.88 \\
\hline$P_{B} \ell$ & $\alpha=0.089$ & $\beta=0.097$ & 0.01 & 0.03 & 0.97 & 0.97 \\
& & & & & & \\
\hline
\end{tabular}

The dashed lines are the cumulative measurements obtained from the literature [13]-[15],[20], [21]. The continuous lines are the cumulative measurements obtained from the CDF model, $P_{B}(\ell)$, adjusted by the least squares method to find the best value for the parameters $\alpha$ and $\beta$, 
considering $\alpha, \beta>0$. The adjusted curve $P_{B}(\ell)$ is plotted along with measured data found in the literature. The other lines are the Exponential, Log-normal, Pareto and Weibull distributions.

The metrics Sum of Squares due to Error (SSE), Root Mean Square Error (RMSE), R-square (RS) and Adjusted R-square (ARS) were used to compute the difference between the analytic and experimental data. SSE and RMSE values close to zero indicate that the model has a small error component. RS is the square of the correlation between the response and the predicted response values. RS can take any value between 0 and 1 , a value closer to 1 indicates that a large proportion of variance is accounted by the model. The ARS statistics can take on any value less than or equal to 1 , a value closer to 1 indicates a better fit [26].

Tafvelin et al [15], collected data during 20 consecutive days, in April 2006, for a bidirectional traffic on an OC-192 backbone link (10 Gbit/s bit rate). For this link, the author used optical splitters attached to two Endace DAG6 2SE cards. The dashed bold line in Figure 5 illustrates the cumulative distribution of IPv4 packet lengths. Table 1 and Figure 5 summarize the comparison between Tafvelin measurements, $P_{B}(\ell)$ and other distributions.

As one can see in Figure 5, the CDF model, $P_{B}(\ell)$, is the nearest curve of the dashed line, cumulative measurements obtained from Tafvelin et al [15]. This result is confirmed by Table 1 results. SSE and RMSE is very close to zero, the best theoric value is zero. For RS and ARS, the best value is one. In this comparison we obtain 0.97 for both (SSE and RMSE). The numeric and graphically results for $P_{B}(l)$ model presents very good results. For this comparison, the second best results are presented by Weibull distribution. In both, $\alpha$ and $\beta$ are the distribuition parameters adjusted using the Matlab fitting tool [26].

A important point to be noted is the presence of two jumps. The first, near zero, and the second near 1500 (MTU). These two points of rapid growth is present in the collected values, but the Exponential, Log-Normal, Pareto and Weibull distribuitions can not capture both. Only the first one, near zero. This not happen in the proposed model.

\subsection{Rastin measurements}

The paper by Rastin Pries [14] presents measurements taken from an ISP switching center providing access to 250 households. The customers access the wireless LAN from several access points, and then the traffic is multiplexed using an IEEE 802.11a radio link. A comparison between Pries measurements, $P_{B}(\ell)$ and other distributions is summarized in Table 10 and Figure 12.

Table 2. Rastin [14] measurements versus, $P_{B}(\ell)$ and other cumulative distributions.

\begin{tabular}{|l|l|l|l|l|l|l|}
\hline Dist. & Par.1 & Par.2 & SSE & RMSE & RS & ARS \\
\hline Exp & $\lambda=5.65$ & - & 0.85 & 0.22 & - & - \\
\hline Log & $\mu=4 \mathrm{e}^{-}-10$ & $\sigma=10$ & 1,36 & 0.27 & - & - \\
\hline Par & $\alpha=0.36$ & $\beta=0.01$ & 0.27 & 0.13 & 0.66 & 0.64 \\
\hline Wei & $\alpha=0.24$ & $\beta=0.18$ & 0.09 & 0.08 & 0.65 & 0.63 \\
\hline$P_{B} \ell$ & $\alpha=0.086$ & $\beta=0.18$ & 0.03 & 0.05 & 0.9 & 0.89 \\
& & & & & & \\
\hline
\end{tabular}




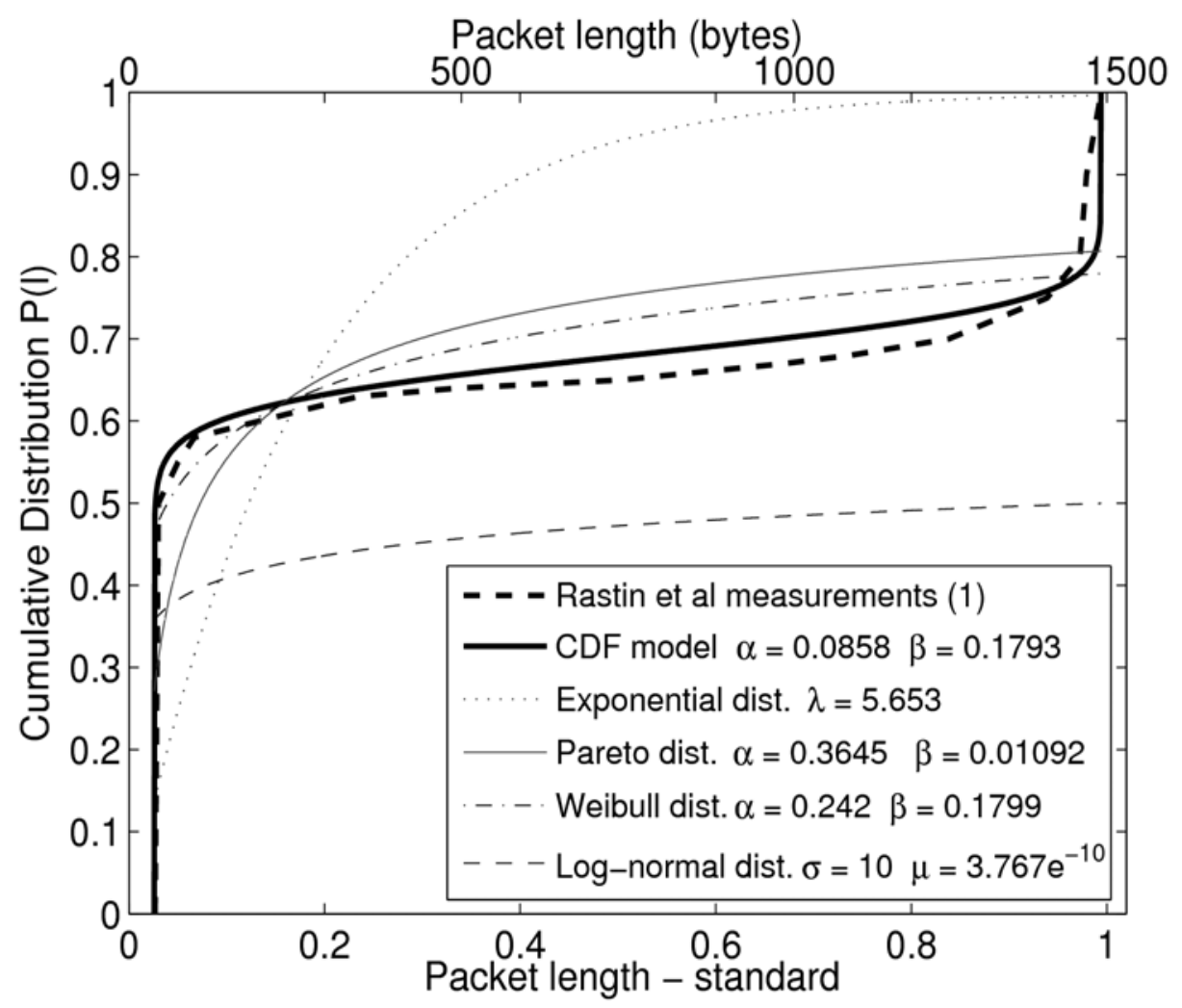

Figure 6. Rastin [14] measurements versus $P_{B}(\ell)$ function and other cumulative distributions.

The Exponential distribution, with $\lambda$ distribuition parameter, presents the worst results in comparsion with Rastin Pries [14] measurements. In Figure 6, the Exponential distribuition is far from the measured values and in Table 2, the SSE and RSME is very distant from zero. For RS and ARS, Matlab fitting tool [26] can not obtain any value. The Log-normal distribuition has same results, $\mu$ and $\sigma$ are the distribuition parameters adjusted using the Matlab fitting tool [26]. The difference is that Exponential is very far for up values and Log-normal is very far for down values. This results confirm that Exponential distribution can not modeled computers packts traffic.

\subsection{CAIDA measurements}

The comparison shown in Figure 7 is from the Cooperative Association for Internet Data Analysis (CAIDA), the measurements were taken from an OC-192 IP backbone link, in march 2008, a link between Chicago and Seattle [21]. Figure 7 and Table 3 summarize the comparison between the CAIDA measurements, $P_{B}(\ell)$ and other distributions. The comparison results show a good fit between the measured values and the $P_{B}(\ell)$ curve.

The Pareto and Weibull distributions, both with $\alpha$ and $\beta$ parameters adjusted using the Matlab fitting tool [26], presents better results than Exponential and Log-Normal distribution in comparsion with CAIDA [21] measurements. In Figure 7, the Pareto and Weibull distributions are very near between then and both have a small distance from the dash-line. In Table3, values of SSE and RSME are small and near zero as expected. For RS and ARS values are near 1. 
International Journal of Computer Networks \& Communications (IJCNC) Vol.5, No.3, May 2013

Table 3. CAIDA [21] measurements versus, $P_{B}(\ell)$ and other cumulative distributions.

\begin{tabular}{|l|l|l|l|l|l|l|}
\hline Dist. & Par.1 & Par.2 & SSE & RMSE & RS & ARS \\
\hline Exp & $\lambda=2.03$ & - & 0.82 & 0.21 & 0.03 & 0.03 \\
\hline Log & $\mu=3 \mathrm{e}^{-12}$ & $\sigma=9.28$ & 0.79 & 0.20 & 0.06 & 0.06 \\
\hline Par & $\alpha=0.21$ & $\beta=0.004$ & 0.08 & 0.07 & 0.76 & 0.75 \\
\hline Wei & $\alpha=0.29$ & $\beta=0.55$ & 0.07 & 0.06 & 0.79 & 0.79 \\
\hline$P_{B} \ell$ & $\alpha=0.10$ & $\beta=0.13$ & 0.03 & 0.04 & 0.95 & 0.94 \\
\end{tabular}

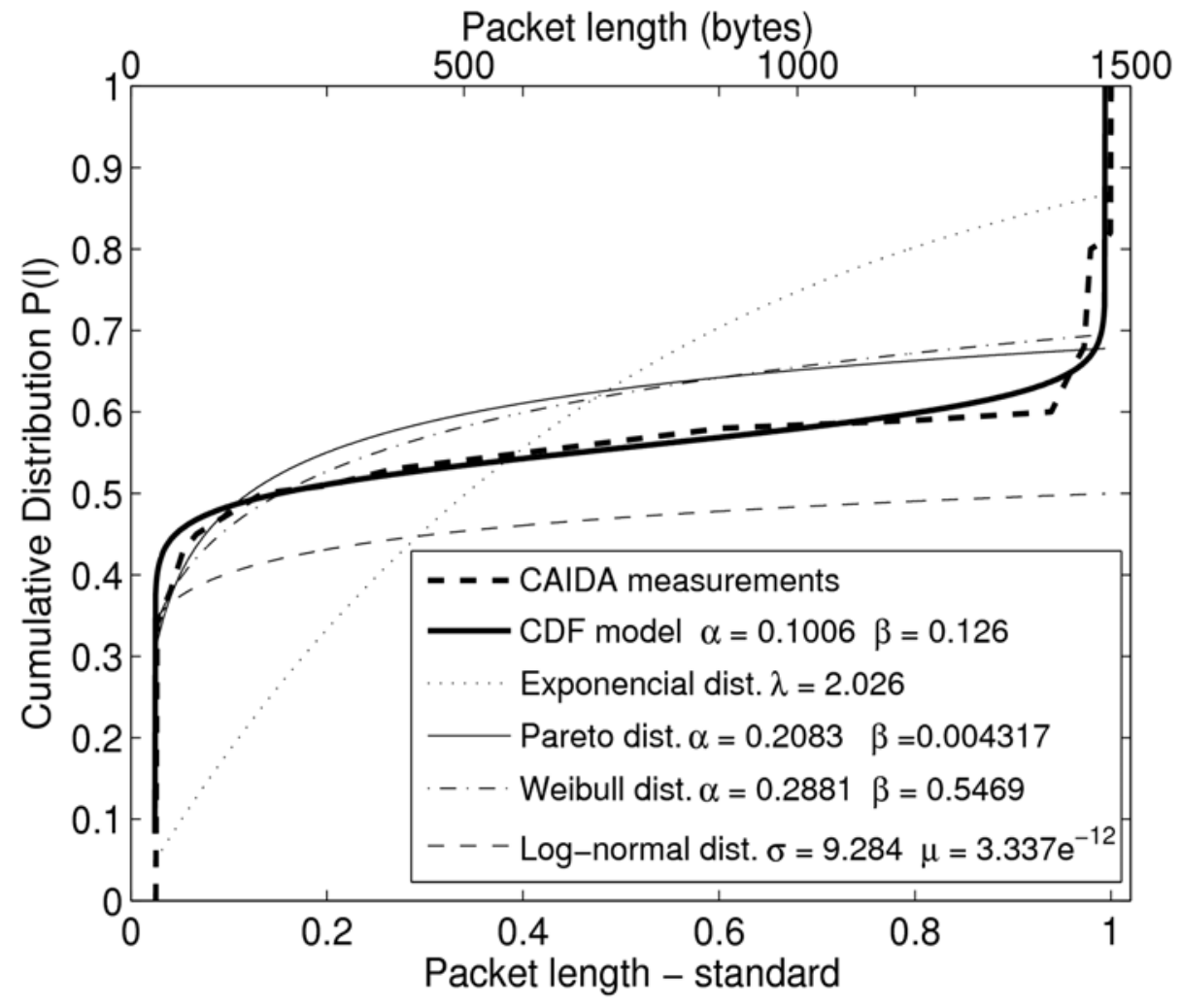

Figure 7. CAIDA [21] measurements versus $P_{B}(\ell)$ function and other cumulative distributions.

\subsection{Sprint measurements}

The Sprint (Sprint Academic Research Group) measurements were collected, in February 2004, from the San Jose $84 \mathrm{Mbit} / \mathrm{s}$ IP backbone link [20]. Sprint measurements, $P_{B}(\ell)$ and other distributions are summarized in Figure 8 and Table 4. The first Sprint set of measurements indicates that $P_{B}(\ell)$ has better results then other cumulative distributions.

For Sprint measurements [20], Exponential and Log-Normal distributions are far from the measured values, as presented in Figure 8 and in Table 4. Pareto and Weibull distributions are better. These explain why both are used in computers traffic-analysis. But for this comparison, 
using the proposed model $P_{B}(\ell)$, the results is improved and this new distribution can be used to modeled computers packts traffic.

Table 4. Sprint [20] measurements versus, $P_{B}(\ell)$ and other cumulative distributions.

\begin{tabular}{|l|l|l|l|l|l|l|}
\hline Dist. & Par.1 & Par.2 & SSE & RMSE & RS & ARS \\
\hline Exp & $\Lambda=3.3$ & - & 0.84 & 0.19 & 0.14 & 0.14 \\
\hline Log & $\mu=1.5 \mathrm{e}-10$ & $\sigma=23.2$ & 1.45 & 0.26 & - & - \\
\hline Par & $\alpha=0.29$ & $\beta=0.006$ & 0.05 & 0.05 & 0.87 & 0.86 \\
\hline Wei & $\alpha=0.32$ & $\beta=0.24$ & 0.05 & 0.05 & 0.88 & 0.88 \\
\hline$P_{B} \ell$ & $\alpha=0.11$ & $\beta=0.21$ & 0.04 & 0.04 & 0.92 & 0.92 \\
& & & & & & \\
\hline
\end{tabular}

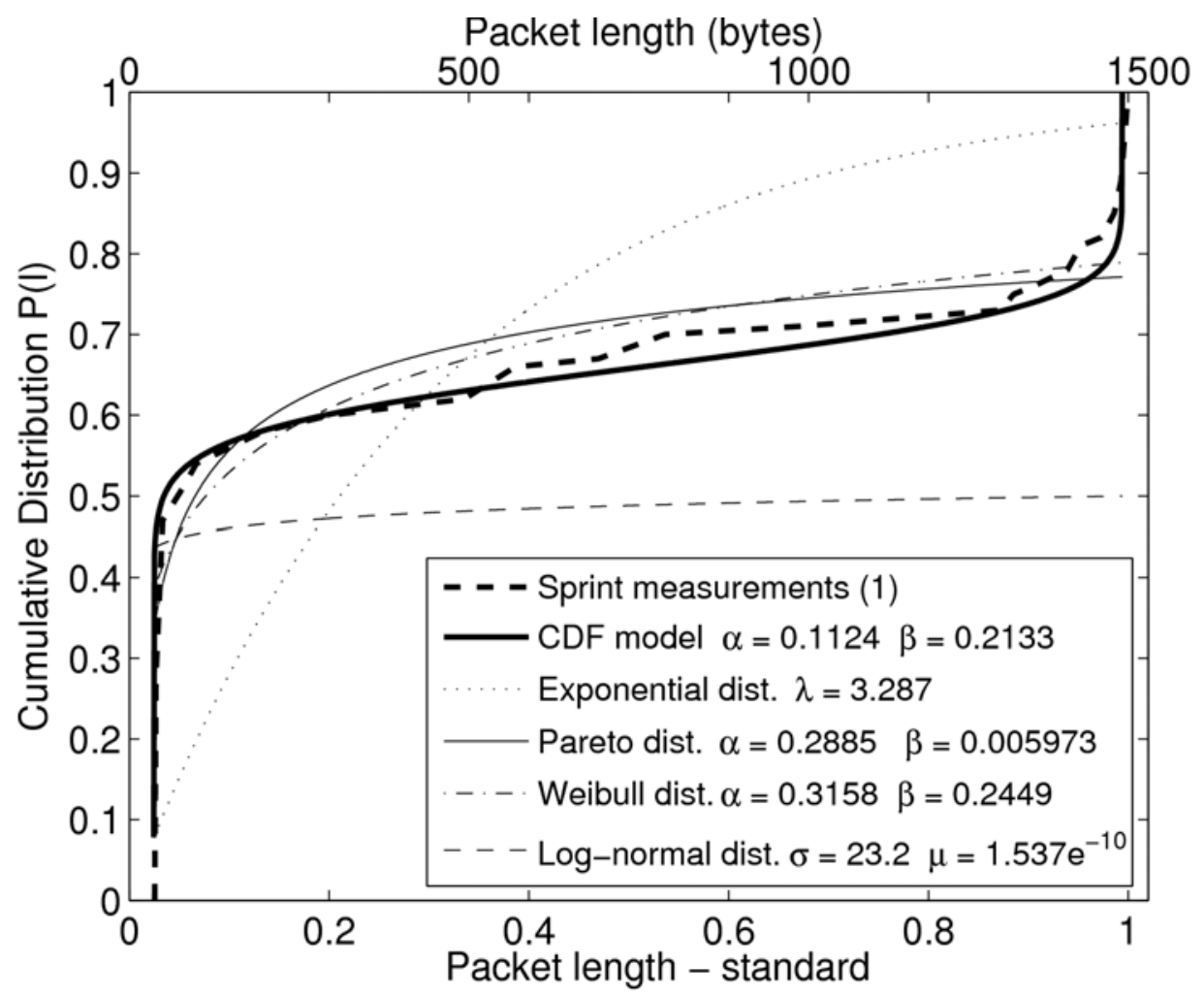

Figure 8. Sprint [20] measurements versus $P_{B}(l)$ function and other cumulative distributions.

\section{Conclusion}

The analysis of the results suggests that the models can be used to estimate the distribution of packet traffic on computer networks. This important information can be used as part of a strategy of traffic identification, application level traffic classification and performance measurement of the routers. Future work in this direction is expected. Besides, the numerical results show that $P_{B}(\ell)$ has a good fitting and that it performs better then the Exponential, Log-normal, Pareto or Weibull distributions, for bimodal traffic. This result is important because one of these distributions may be used in network traffic simulators. 
Two different equations were also obtained to model the packet length probability density function using the Matlab fitting tool [26]. The equations use a sum of two Exponential two Normal distributions. The disadvantage is that, with the Exponential, four parameters are needed to adjust, and the use of Normal distribution implies six parameters to adjust. In both cases, the results are better then $p_{B}(\ell)$, but the increase in the number of parameters, makes it more difficult to associate the measured parameters and the variables present in the computer network traffic.

Some new possible application for the proposed cumulative distributions are improve the metrics for measuring the performance of routers, strategy to be used in the characterization of Internet traffic and help to discover denial-of-service (DoS) attacks characteristics. In papers [27] and [28], some preliminary results and models are presented.

It was also found that many of the measurements made on $10 \mathrm{Gbit} / \mathrm{s}$ (OC-192) links made by [15], [21], [20] and for the access network by [14] used cumulative packet length data. Thus, this paper also presented a cumulative distribution to compare the measured data with the proposed model.

As suggestions for future work, it is possible to perform more measurements of packet lengths in the network layer for other specific applications (Skype, games on-line, etc) to classify the traffic based on the values of $\alpha$ and $\beta$ of the new proposed distribution. The traffic classification, based on the packet lengths has already been done by some other authors in recent papers [1]--[5].

\section{REFERENCES}

[1] Jaber, M. and Cascella, R. G. and Barakat, C., Using host profiling to refine statistical application identification, Proceedings of the IEEE INFOCOM 2012, 2746--2750, 2012.

[2] Rocha, E. and Salvador, P. and Nogueira, A., Detection of Illicit Network Activities based on Multivariate Gaussian Fitting of Multi-Scale Traffic Characteristics, Proceedings of the IEEE ICC 2011, 1-$6,2011$.

[3] Jaber, M. and Cascella, R. G. and Barakat, C., Can we trust the inter-packet time for traffic classification?, Proceedings of the IEEE ICC 2011, 1--5, 2011.

[4] Dainotti, A. and Pescape, A. and Kim, Hyun-chul, Traffic Classification through Joint Distributions of Packet-level Statistics, Proceedings of the IEEE GLOBECOM 2011, 1--6, 2011.

[5] Wen Zhang, Peer-to-Peer Traffic Anti-identification Based on Packet Size, Proceedings of the IEEE International Conference on Computer Science and Network Technology 2011, 2277--2280, 2011.

[6] Callado, A. and Kamienski, C. and Szabo, G. and Gero, B. and Kelner, J. and Fernandes, S. and Sadok, D., A Survey on $\backslash \mathrm{rm}\{\mathrm{I}\}$ nternet Traffic Identification, IEEE Communications Surveys Tutorials, 11, 37--52, 2009.

[7] Alfonso Iacovazzi and Andrea Baiocchi, Optimum packet length masking, 22nd International Teletraffic Congress (ITC), 2010, Amsterdam, The Netherlands, 7-9, September, 2010.

[8] Thomas Karagiannis and Andre Broibo and Nevil Brownlee and K. C. Claffy and Michalis Faloutsos, File-sharing in the Internet: A characterization of P2P traffic in the backbone, University of California, Riverside, USA, CA 92521, Tech. Rep., November, 2003.

[9] F. Liu and Z. Li and J. Yu, P2P Applications Identification Based On the Statistics Analysis of Packet Length, IEEE International Symposium on Information Engineering and Electronic Commerce, 160--163, 2009.

[10] D. Pan and Y. Yang, Localized Independent Packet Scheduling for Buffered Crossbar Switches, IEEE Transactions on Computers, 58, 260--274, February, 2009.

[11] Z. Cucej and M. Fras, Data Source Statistics Modeling based on Measured Packet Traffic: A Case Study of Protocol Algorithm and Analytical Transformation Approach, IEEE International Conference on Telecommunications in Modern Satellite, Cable and Broadcasting Services, TELSIKS 2009, 54--64, 2009. 
[12] N. Duffield and C. Lund and M. Thorup, Learn More, Sample Less: Control of Volume and Variance in Network Measurement, IEEE Transactions on Information Theory, 51, 1756--1775, May, 2005.

[13] Cheng Yu and V. Ravindran and A. Leon-Garcia, Internet Traffic Characterization Using Packet-Pair Probing, 26th IEEE International Conference on Computer Communications. INFOCOM 2007, 1766-1774, 2007.

[14] Rastin Pries and F. Wamser and D. Staehle and P. Tran-Gia, Traffic Measurement and Analysis of a Broadband Wireless Internet Access, IEEE 69th Vehicular Technology Conference. VTC Spring, 1--5, April, 2009.

[15] W. John and S. Tafvelin, Analysis of Internet Backbone Traffic and Header Anomalies Observed, IMC '07: Proceedings of the 7th ACM SIGCOMM conference on Internet measurement, New York, NY, USA, 111--116, 2007.

[16] R. Beverly and K. C. Claffy, Wide-area IP multicast traffic characterization, IEEE Network, 17, 8--15, 2003.

[17] Andrej Kos and Matevz Pustisek and Janez Bester, Characteristics of real packet traffic captured at different network locations, Computer as a Tool. The IEEE Region 8 EUROCON, 1, 164--168, 2003.

[18] Li Bo and David J. Parish and J. M. Sandford, Using TCP Packet Size Distributions for Application Detection, The 7th Annual PostGraduate Symposium on The Convergence of Telecommunications, Networking and Broadcasting, 2006.

[19] Rishi Sinha and Christos Papadopoulos and John Heidemann, Internet Packet Size Distributions: Some Observations, USC/Information Sciences Institute, 2007.

[20] Sprintlabs, Sprint Academic Research Group -- Packet Size Distribution, 2004. Access in Jun 2010, https://research.sprintlabs.com/packstat/packetoverview.php

[21] The Cooperative Association for Internet Data Analysis -- Packet size distribution comparison between Internet links in 1998 and 2008, 2008. Access in October 2010,www.caida.org/research/trafficanalysis/pkt\size $\backslash$ distribution/graphs.xml

[22] Sajjad Âli Mushtaq and Azhar A. Rizvi, Statistical analysis and mathematical modeling of network (segment) traffic, Proceedings of the IEEE Symposium on Emerging Technologies, 246--251, September, 2005 .

[23] Sean McCreary and K. C. Claffy, Trends in Wide Area IP Traffic Patterns -- A View from Ames Internet Exchange, Proceedings of the 13th ITC Specialist Seminar on Internet Traffic Measurement and Modeling, Monterey, CA, 2000.

[24] Ville Mattila, Traffic Analysis -- A review of Internet traffic packet size distributions, 2010. Accessed

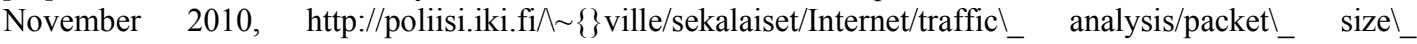
distribution

[25] RFC-1042, A Standard for the Transmission of $\backslash \mathrm{rm}\{$ IP $\}$ Datagrams over IEEE 802 Networks, February, 1988. Acess in October 2010.http://www.faqs.org/rfcs/rfc1042.html .

[26] Matlab, Matlab -- The Language of Technical Computing, http://www.mathworks.com/products/matlab/, 2010, Accessed October 2010.

[27] Ewerton Castro and Iguatemi E. Fonseca and Ajey Kumar and Marcelo S. Alencar, A Packet Distribution Traffic Model for Computer Networks, The International Telecommunications Symposium, ITS 2010, Manaus, Brazil. September, 2010.

[28] Ewerton Castro and Iguatemi E. Fonseca and Marcelo S. Alencar, Comparison Results of a Mathematical Model and Experimental Measurements for the Distribution Function of the Packet Length in Computer Networks, International Workshop on Telecommunications 2011 -- IWT 2011.

[29] Ewerton Castro and Iguatemi E. Fonseca and Ajey Kumar and Marcelo S. Alencar, A New Packet Distribution Model for Computer Networks with Bimodal Traffic, Journal of Communication and Computer, pg 208--216, number 2, 2012, ISSN 1548-7709.

[30] Ewerton Romulo Silva Castro, Modelo para a Distribuição de Probabilidade do Comprimento dos Pacotes em Redes de Computadores, Phd Thesis, UFCG, Campina Grande, PB. Brazil, 2011. 